\title{
Natural Metric for Quantum Information Theory
}

\author{
P.W. Lamberti ${ }^{1}$, M. Portesi ${ }^{2}$ and J. Sparacino ${ }^{1}$ \\ 1 Facultad de Matemática, Astronomía y Física, \\ Universidad Nacional de Córdoba and CONICET, Ciudad Universitaria, 5000 Córdoba, Argentina \\ 2 Instituto de Física La Plata (CONICET) and Departamento de Física, \\ Facultad de Ciencias Exactas, Universidad Nacional de La Plata, CC 67, 1900 La Plata, Argentina
}

(Dated: November 4, 2018)

\begin{abstract}
We study in detail a very natural metric for quantum states. This new proposal has two basic ingredients: entropy and purification. The metric for two mixed states is defined as the square root of the entropy of the average of representative purifications of those states. Some basic properties are analyzed and its relation with other distances is investigated. As an illustrative application, the proposed metric is evaluated for 1-qubit mixed states.
\end{abstract}

PACS numbers: 03.67.-a; 03.67.Mn; 03.65.-w

\section{INTRODUCTION}

The study of distances and metrics between quantum states is a topic of permanent interest, which has been lately rekindled on account of problems emerging in quantum information theory (QIT) 1]. Distances are used as measure of distinguishability between quantum states [2] and in the definition of the degree of entanglement [3], just to mention two very relevant examples. They also characterize the geometrical structure of the space of quantum states [4].

In the mathematical formalism of quantum mechanics the states of a physical system $\mathcal{S}$ are represented by operators (density operators) acting on a Hilbert space $\mathcal{H}$. More precisely the states of the system $\mathcal{S}$ are represented by the elements of $\mathcal{B}(\mathcal{H})_{1}^{+}$, that is, the set of positive, trace-one operators on $\mathcal{H}$. The notion of a state as a unit vector of $\mathcal{H}$ refers to the extremal elements of $\mathcal{B}(\mathcal{H})_{1}^{+}\left(\rho \in \mathcal{B}(\mathcal{H})_{1}^{+}\right.$is extremal if and only if it is idempotent, $\left.\rho^{2}=\rho\right)$. In this case $\rho$ is of the form $|\psi\rangle\langle\psi|$ for some unit vector $|\psi\rangle \in \mathcal{H}$, and it is called a pure state. If $\rho$ is not idempotent, the corresponding state is called mixed.

In introducing distances between quantum states different roads have been traversed [5, 6, 7, 8, 9, 10, 11. For example Wootters arrived at the distance

$$
d_{W}(|\psi\rangle,|\varphi\rangle)=\arccos (|\langle\psi \mid \varphi\rangle|)
$$

by analyzing the statistical fluctuations in the outcomes of measurements into the quantum mechanics formalism [5]. In Eq. (10), $\langle\psi \mid \varphi\rangle$ represents the inner product between the pure states $|\psi\rangle$ and $|\varphi\rangle$, and therefore $d_{W}$ gives the "angle" between these two states.

Another way of dealing with the problem of introducing distances between quantum states is to generalize the notions of distance defined in the space of classical probability distributions. This is the case of the relative entropy, which is a generalization of information theoretic Kullback-Leibler divergence. The relative entropy of an operator $\rho$ with respect to an operator $\sigma$, both belonging to $\mathcal{B}(\mathcal{H})_{1}^{+}$, is

$$
S(\rho, \sigma)=\operatorname{Tr}\left[\rho\left(\log _{2} \rho-\log _{2} \sigma\right)\right]
$$

where $\log _{2}$ stands for logarithm in base two. The relative entropy is not a metric (because it is not symmetric and does not verify the triangle inequality). Even worst, it may even be unbounded. In particular, the relative entropy is well defined only when the support of $\sigma$ is equal to or larger than that of $\rho$ [12] (the support of an operator is the subspace spanned by the eigenvectors of the operator with nonzero eigenvalues). This is a strong restriction which is violated in some physically relevant situations, as for example when $\sigma$ is a pure reference state.

Recently we have investigated a distance between mixed quantum states that was named the quantum JensenShannon divergence (QJSD) and that is a symmetrized version of relative entropy (2):

$$
D_{J S}(\rho, \sigma)=\frac{1}{2}\left[S\left(\rho, \frac{\rho+\sigma}{2}\right)+S\left(\sigma, \frac{\rho+\sigma}{2}\right)\right] .
$$

The main properties of the QJSD as a distinguishability measure have been presented in Ref. 13]; the metric character of the square root of $D_{J S}$ has also been reported recently [14, 15]. It has several interesting interpretations into the realm of QIT and it has been applied as a measure of the degree of entanglement 16].

In this work we propose an alternative metric between quantum states, that we think, from a conceptual point of view, is a very natural one and which is, in some sense, a derivative of the QJSD. This proposal hinges on two central 
concepts of QIT: entropy and purification. Relevant properties of these notions are quoted in the following section. The rest of the paper is organized as follows: in Section II we consider a measure of distance for pure states defined using von Neumann entropy and we study its properties. Section IV is devoted to the new proposal of a distance between mixed states by recourse to the concept of purification. We then present in Section $\nabla$ an application for the case of 1-qubit mixed states with a numerical example. Finally, concluding remarks are drawn in Section VI

\section{SOME COMMENTS ON ENTROPY AND PURIFICATION}

Entropy is a fundamental notion in classical and in quantum information theory. In the classical case most of the results concerning the coding problem can be expressed in terms of the Shannon entropy

$$
H_{S}(P)=-\sum_{i} p_{i} \log _{2} p_{i}
$$

where $P=\left\{p_{i} \geq 0, \sum_{i} p_{i}=1\right\}$ is a (discrete) probability distribution [17]. In the quantum context the expression for entropy differs from the Shannon entropy. For a mixed state described by the density operator $\rho$, von Neumann defined the entropy of $\rho$ as

$$
H_{N}(\rho)=-\operatorname{Tr}\left(\rho \log _{2} \rho\right)=-\sum_{i} \lambda_{i} \log _{2} \lambda_{i}
$$

with $\left\{\lambda_{i}\right\}$ being the set of eigenvalues of the operator $\rho$.

The von Neumann entropy has several interesting properties [18]. Among them we remark the one that gives an upper bound for a convex combination $\rho=\sum_{i} p_{i} \rho_{i} \quad\left(\right.$ with $\left.\sum_{i} p_{i}=1\right)$ :

$$
H_{N}(\rho) \leq \sum_{i} p_{i} H_{N}\left(\rho_{i}\right)+H_{S}\left(\left\{p_{i}\right\}\right) .
$$

Equality is verified if and only if the states $\rho_{i}$ have supports in orthogonal subspaces.

Although expressions (4) and (5) look similar, they are quite different. These differences are particularly relevant for quantum information theory. For example, if $a$ is a message taken from the source $\mathcal{A}$ and $p(a)$ is the probability of the message $a$, the Shannon entropy of the source is

$$
H_{S}[\mathcal{A}]=-\sum_{a} p(a) \log _{2} p(a)
$$

Let us now suppose that we have a quantum signal source, that is a device that codes a message $a$ taken from the source $\mathcal{A}$ into a signal state $\left|a_{S}\right\rangle$ of a quantum system $\mathcal{S}$. The ensemble of signals from the signal source will be represented by the density operator

$$
\mu=\sum_{a} p(a)\left|a_{S}\right\rangle\left\langle a_{S}\right| .
$$

Then, if the signal states $\left|a_{S}\right\rangle$ are not orthogonal and from (6), the inequality

$$
H_{N}(\mu)<H_{S}(\{p(a)\})
$$

is satisfied. The physical consequences of this inequality have been analyzed in detail by Jozsa and Schlienz [19].

Another important property of von Neumann entropy is that it gives the number of qubits necessary to represent a quantum signal faithfully. Indeed, let us suppose that Alice has a source of pure qubit signal states $|\psi\rangle$ and $|\varphi\rangle$. Each emission is chosen to be $|\psi\rangle$ or $|\varphi\rangle$ with an equal prior probability one half. Then in this case the density matrix of the source is $\pi=\frac{1}{2}(|\psi\rangle\langle\psi|+| \varphi\rangle\langle\varphi|)$. Alice may communicate the sequence of states to Bob by transmitting one qubit per emitted state. But, according to the quantum source coding theorem, the quantity

$$
H_{N}\left(\frac{|\psi\rangle\langle\psi|+| \varphi\rangle\langle\varphi|}{2}\right)
$$

gives the lowest number of qubits per state that Alice needs to communicate the quantum information (with arbitrarily high fidelity) 20]. 
Purification is the second key concept we use in proposing a metric for quantum states. It has to do with the fact that every mixed quantum state can be interpreted as being part of a higher-dimensional pure state. From a physical point of view the notion of purification provides, for example, insight into the mechanism of quantum decoherence 21]. Formally it can be described in the following way: let $\rho$ be any mixed state on the Hilbert space $\mathcal{H}$. A purification of $\rho$ is any pure state $|\psi\rangle$ in any extended Hilbert space $\mathcal{H} \otimes \mathcal{H}_{\text {aux }}$ with the property that

$$
\rho=\operatorname{Tr}_{a u x}|\psi\rangle\langle\psi|
$$

where $\operatorname{Tr}_{a u x}$ stands for the partial trace on the Hilbert space $\mathcal{H}_{a u x}$. In other words a purification is any pure state having $\rho$ as the reduced state for subsystem [8].

If $\rho$ admits the decomposition $\rho=\sum_{i} p_{i}\left|e_{i}\right\rangle\left\langle e_{i}\right|$ where $p_{i} \geq 0$ and $\sum_{i} p_{i}=1$, an example for a purification of $\rho$ is given by

$$
|\psi\rangle=\sum_{i} \sqrt{p_{i}}\left|e_{i}\right\rangle \otimes\left|a_{i}\right\rangle
$$

with the auxiliary states $\left|a_{i}\right\rangle$ being mutually orthogonal.

We complete this section by remarking two facts on purifications that will be used later on:

1. It can be shown that for two purifications $\left|\psi_{1}\right\rangle$ and $\left|\psi_{2}\right\rangle$ of the state $\rho$, there exists a unitary transformation $U$ acting on $\mathcal{H}_{\text {aux }}$ such that

$$
\left|\psi_{1}\right\rangle=(I \otimes U)\left|\psi_{2}\right\rangle
$$

where $I$ is the identity operator on the space $\mathcal{H}[1]$.

2. If one is interested in purifications of two states it can be assumed, without loss of generality, that the purifications lie in the same extended Hilbert space [8].

\section{VON NEUMANN ENTROPY AS A METRIC FOR PURE STATES}

Let $|\psi\rangle$ and $|\varphi\rangle$ be two pure states in a given Hilbert space $\mathcal{H}$. We define the distance between these two states in the form:

$$
D_{N}(|\psi\rangle,|\varphi\rangle) \equiv \sqrt{H_{N}\left(\frac{|\psi\rangle\langle\psi|+| \varphi\rangle\langle\varphi|}{2}\right)}
$$

It should be noted that this expression corresponds to the square root of the QJSD when evaluated between pure states. Indeed, in terms of the von Neumann entropy (5), the QJSD (3) can be rewritten in the form:

$$
D_{J S}(\rho, \sigma)=H_{N}\left(\frac{\rho+\sigma}{2}\right)-\frac{1}{2} H_{N}(\rho)-\frac{1}{2} H_{N}(\sigma)
$$

But for a pure state the von Neumann entropy vanishes; then for the corresponding operators $\rho=|\psi\rangle\langle\psi|$ and $\sigma=|\varphi\rangle\langle\varphi|$ it results:

$$
D_{J S}(|\psi\rangle\langle\psi|,| \varphi\rangle\langle\varphi|)=D_{N}^{2}(|\psi\rangle,|\varphi\rangle)
$$

After some algebra, we can rewrite the square of Eq. 111, in terms of the inner product between $|\psi\rangle$ and $|\varphi\rangle$ :

$$
D_{N}^{2}(|\psi\rangle,|\varphi\rangle)=\Phi(|\langle\psi \mid \varphi\rangle|)
$$

where

$$
\Phi(x) \equiv-\left(\frac{1-x}{2}\right) \log _{2}\left(\frac{1-x}{2}\right)-\left(\frac{1+x}{2}\right) \log _{2}\left(\frac{1+x}{2}\right), \quad 0 \leq x \leq 1
$$

The main properties of $D_{N}(|\psi\rangle,|\varphi\rangle)$ given by Eq. (11) are:

- It is symmetric. 
- It vanishes if and only if $|\psi\rangle=e^{i \alpha}|\varphi\rangle \quad$ (i.e., both states belong to the same ray).

- It is bounded: $0 \leq D_{N}(|\psi\rangle,|\varphi\rangle) \leq 1$ (this can be easily verified from inequality (6) and from the fact that the von Neumann entropy vanishes for a pure state).

- It verifies the triangle inequality (but $H_{N}\left(\frac{|\psi\rangle\langle\psi|+| \varphi\rangle\langle\varphi|}{2}\right)$ does not) [14, 15].

All these properties give $D_{N}$ the metric character.

The distance $D_{N}$ between two neighboring pure states $|\psi\rangle=\sum_{j} \sqrt{p_{j}} e^{i \phi_{j}}|j\rangle$ and $|\tilde{\psi}\rangle=|\psi\rangle+|d \psi\rangle=$ $\sum_{j} \sqrt{p_{j}+d p_{j}} e^{i\left(\phi_{j}+d \phi_{j}\right)}|j\rangle$, where $\{|j\rangle\}$ in an orthonormal basis, is given by:

$$
D_{N}^{2}(|\psi\rangle,|\tilde{\psi}\rangle) \simeq \frac{1}{8} \sum_{j} \frac{d p_{j}^{2}}{p_{j}}
$$

From expression (11) it is easily checked that, up to second order in $d p_{j}$, the following relation exists between Wootters distance and the distance $D_{N}$ given in (11):

$$
d_{W}^{2}(|\psi\rangle,|\tilde{\psi}\rangle)=2 D_{N}(|\psi\rangle,|\tilde{\psi}\rangle)
$$

A similar relation is also true for the Fubini-Study metric 22].

\section{EXTENSION OF THE DEFINITION OF THE METRIC TO MIXED STATES}

In the previous section we have thought the square root of the von Neumann entropy of the average $\frac{1}{2}(|\psi\rangle\langle\psi|+| \varphi\rangle\langle\varphi|)$ as a true metric between two pure states $|\psi\rangle$ and $|\varphi\rangle$. Here we investigate its extension to mixed states.

We start by recalling that the Bures distance between two mixed states, that is for two operators $\rho$ and $\sigma$ belonging to $\mathcal{B}(\mathcal{H})_{1}^{+}$, is given by

$$
D_{B}(\rho, \sigma)=\sqrt{2-2 F(\rho, \sigma)}
$$

where $F(\rho, \sigma)=\operatorname{Tr} \sqrt{\rho^{1 / 2} \sigma \rho^{1 / 2}}$ is the fidelity function (see, for instance, Ref. [1]). A well-known result (Uhlmann's theorem [7, [] ) asserts that the fidelity $F(\rho, \sigma)$ can be expressed in the form

$$
F(\rho, \sigma)=\max _{|\psi\rangle,|\varphi\rangle}|\langle\psi \mid \varphi\rangle|
$$

where the maximization is performed over all purifications $|\psi\rangle$ of $\rho$ and all purifications $|\varphi\rangle$ of $\sigma$. Then, the Bures metric can be rewritten in the form:

$$
D_{B}(\rho, \sigma)=\min _{|\psi\rangle,|\varphi\rangle} \sqrt{2-2|\langle\psi \mid \varphi\rangle|}
$$

where the minimum is taken over all purifications of $\rho$ and $\sigma$.

By mimicking this last expression, we can define from Eq. (11), a metric for arbitrary mixed states $\rho$ and $\sigma$ belonging to $\mathcal{B}(\mathcal{H})_{1}^{+}$:

$$
D_{N}(\rho, \sigma) \equiv \min _{|\psi\rangle,|\varphi\rangle} \sqrt{H_{N}\left(\frac{|\psi\rangle\langle\psi|+| \varphi\rangle\langle\varphi|}{2}\right)}
$$

where, once again, the minimum is taken over all purifications $|\psi\rangle$ of $\rho$ and all purifications $|\varphi\rangle$ of $\sigma$. Recalling Eq. (12) and due to the decreasing nature of $\Phi(x)$ as a function of $x=|\langle\psi \mid \varphi\rangle| \in[0,1]$, Eq. (13), to seek the minimum out in our proposal (17) is equivalent to look for the purifications that maximize the overlap $|\langle\psi \mid \varphi\rangle|$. Clearly, by construction, the metric character of $D_{N}(\rho, \sigma)$ is kept for mixed states. It also is always well defined.

From a conceptual point of view, our proposal is equivalent to replace the problem of measuring the distance between two arbitrary mixed states, $\rho$ and $\sigma$, by the problem of distinguishability of all the ensembles $\mathcal{E}=\left\{|\psi\rangle,|\varphi\rangle ; p_{1}=\frac{1}{2}, p_{2}=\right.$ $\left.\frac{1}{2}\right\}$ built from purifications of $\rho$ and $\sigma$ respectively [19]. This is the central point in our proposal.

According to identity (15), the metric $D_{N}(\rho, \sigma)$ can be expressed in terms of the fidelity in the form:

$$
D_{N}(\rho, \sigma)=\sqrt{\Phi(F(\rho, \sigma))}
$$

By using this representation, we can derive the main properties of the metric $D_{N}(\rho, \sigma)$. For example 
- $D_{N}$ is invariant under unitary transformations: $D_{N}\left(U \rho U^{\dagger}, U \sigma U^{\dagger}\right)=D_{N}(\rho, \sigma)$.

- If $\mathcal{Q}$ is a trace-preserving quantum operation, then: $D_{N}(\mathcal{Q}(\rho), \mathcal{Q}(\sigma)) \leq D_{N}(\rho, \sigma)$.

As a textbook exercise we can derive an alternative expression for fidelity [1]:

$$
F(\rho, \sigma)=\max _{|\varphi\rangle}|\langle\psi \mid \varphi\rangle|
$$

where $|\psi\rangle$ is any fixed purification of $\rho$, and the maximization is performed over all purifications $|\varphi\rangle$ of $\sigma$. Therefore, by using this expression and Eq. (18), the new metric (17) can be reexpressed in a more convenient way:

$$
D_{N}(\rho, \sigma)=\min _{|\varphi\rangle} \sqrt{H_{N}\left(\frac{|\psi\rangle\langle\psi|+| \varphi\rangle\langle\varphi|}{2}\right)}
$$

where $|\psi\rangle$ is any fixed purification of $\rho$, and the minimization is taken over all purifications $|\varphi\rangle$ of $\sigma$.

Several interesting questions arise from the definition of distance between quantum states given by Eq. (177). Among them we remark the following two:

- The first one has to do with the computability of $D_{N}(\rho, \sigma)$. As it was said, the purifications of $\rho$ and $\sigma$ can be thought as belonging to the same extended Hilbert space. Furthermore, according to Eq. (10), every purification is related to another one by a unitary transformation. Therefore the technical task of finding the minimum in Eq. (20) is equivalent to finding unitary operations acting on the auxiliary Hilbert space that maximize the overlap $|\langle\psi \mid \varphi\rangle|$. This point will be analyzed, for a particular example, in the next section.

- The second point to be remarked is the relevance of the metric $D_{N}(\rho, \sigma)$ to the light of decoherence. Decoherence can be viewed as the unitary evolution of a compound system consisting of the system itself and its environment, under which both components become entangled 21]. In this context, the auxiliary space $\mathcal{H}_{\text {aux }}$ corresponds to the environment.

\section{EVALUATION OF THE METRIC $D_{N}$ FOR 1-QUBIT MIXED STATES}

As an illustrative application, we now evaluate the metric $D_{N}$ given in Eq. (17), for two 1-qubit states. In this case it is convenient to use the Bloch representation for a mixed 1-qubit state:

$$
\rho=\frac{I_{2}+\vec{r} \cdot \vec{\sigma}}{2}
$$

where $I_{n}$ denotes the $n \times n$ identity matrix, the Bloch vector $\vec{r}$ is a 3 -dimensional vector such that $\|\vec{r}\| \leq 1$, and the vector $\vec{\sigma}$ has as components the Pauli matrices. The matrix $\rho$ belongs to the complex plane $C^{2}$. In Ref. 23] it is shown that a purification of an arbitrary density $\rho$ acting on $C^{2} \otimes C^{2}$ (that is, a $4 \times 4$ matrix) is given by

$$
P_{\rho}=\frac{1}{2}\left(I_{4}+\sum_{i} r_{i} \sigma_{i} \otimes I_{2}+\sum_{i} \gamma_{i} I_{2} \otimes \sigma_{i}+\sum_{i, j} A_{i j} \sigma_{i} \otimes \sigma_{j}\right)
$$

where $i, j=1,2,3, \quad r_{i}$ are the components of the vector $\vec{r}, \gamma_{i}$ are the components of the vector $\vec{\gamma}=A^{T} \vec{r}$, and the $3 \times 3$ real matrix $A$ is a solution of the system of equations:

$$
\begin{aligned}
A A^{T} & =(1-\|\vec{r}\|) I_{3}+\vec{r} \vec{r}^{T} \\
\operatorname{det}(A) & =\|\vec{r}\|^{2}-1
\end{aligned}
$$

The result by Constantinescu et al. 23] establishes further that all purification of $\rho$ is of the form (22) with matrix $A=\tilde{A} S$, with $S$ being an element of the Lie group $S O\left(3, \mathbb{R}^{3}\right)$ and $\tilde{A}$ a particular solution of the system (23). Therefore, in this case the evaluation of the minimum in Eq. 20 to obtain $D_{N}$ leads to an extremization problem over the parameter space of the Lie group $S O\left(3, \mathbb{R}^{3}\right)$.

As a particular example, let us show how to find this minimum by evaluating the distance $D_{N}\left(\rho, \mathcal{E}_{p}(\rho)\right)$ between an input state $\rho$ and the state emerging from a depolarizing channel

$$
\mathcal{E}_{p}(\rho)=\frac{p I_{2}}{2}+(1-p) \rho, \quad 0 \leq p \leq 1 .
$$




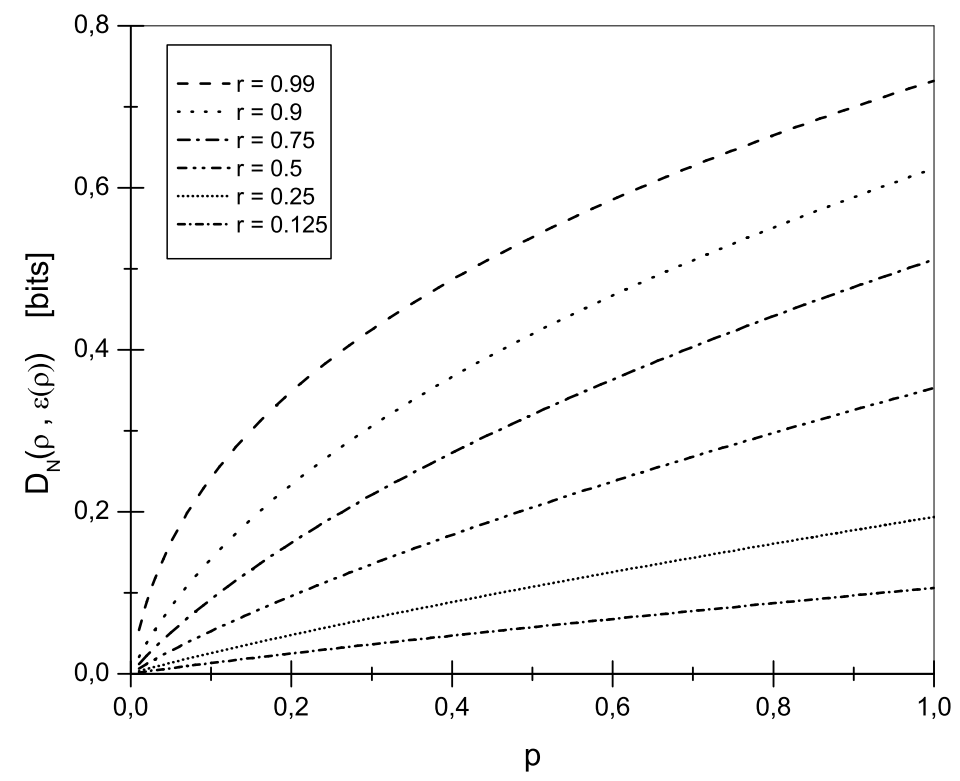

FIG. 1: Values of the distance $D_{N}\left(\rho, \mathcal{E}_{p}(\rho)\right)$ between the state $\rho$ and the emerging ones from the depolarizing channel as a function of $p$, for different values of the norm $r=\|\vec{r}\|$ of the Bloch vector (see Eqs. (21) and (24)).

The depolarizing channel can be viewed as a process that takes a system to a fully mixed state with probability $p$, or leaves it unchanged with probability $1-p$. The effect of the depolarizing channel (24) over the state $\rho$ is to "contract" the vector $\vec{r}$ in the factor $(1-p)$ :

$$
\rho(\vec{r}) \rightarrow \mathcal{E}_{p}(\rho)=\rho\left(\overrightarrow{r^{\prime}}\right), \quad \overrightarrow{r^{\prime}}=(1-p) \vec{r}
$$

A strategy for evaluating the metric $D_{N}\left(\rho, \mathcal{E}_{p}(\rho)\right)$ could be established in the following way: let $A$ be a solution of the system (23) corresponding to a Bloch vector $\vec{r}$, and let $A_{p}$ be the solution of (23) corresponding to $(1-p) \vec{r}$. A simple calculation shows that

$$
\begin{aligned}
A_{p} A_{p}^{T} & =A A^{T}+f(p) \Omega \\
\operatorname{det} A_{p} & =\operatorname{det} A-f(p)\|\vec{r}\|^{2}
\end{aligned}
$$

where $f(p)=1-(1-p)^{2}$ and $\Omega=\|\vec{r}\|^{2} I_{3}-\vec{r} \vec{r}^{T}$. Now we take a fixed purification of $\rho$ characterized by the matrix $A$ (solution of (23)) and evaluate a matrix $\tilde{A}_{p}$ that satisfies (25) (associated with purification $P_{\mathcal{E}_{p}(\rho)}$ ). Then every purification of $\mathcal{E}_{p}(\rho)$ can be expressed in the form $A_{p}=\tilde{A}_{p} S$ with $S$ being an element of $S O\left(3, \mathbb{R}^{3}\right)$. Thus to find $S \in S O\left(3, \mathbb{R}^{3}\right)$ that minimizes our proposal $\left[20\right.$, is equivalent to find $S \in S O\left(3, \mathbb{R}^{3}\right)$ such that minimizes the norm $\left\|\tilde{A}_{p} S-A\right\|_{H S}$ 23]. Here $\|\cdot\|_{H S}$ stands for the Hilbert-Schmidt norm. This is the classical Procrustes problem. An algorithmic solution of the Procrustes problem can be found in Ref. 24]. By solving the systems of equations (23) and (25) and applying the algorithm for solving the Procrustes problem, we can find the purification $P_{\mathcal{E}_{p}(\rho)}$ that extremizes (20). Then by calculating the eigenvalues of the "average" $\frac{P_{\rho}+P_{\mathcal{E}_{p}(\rho)}}{2}$ we can evaluate the distance $D_{N}\left(\rho, \mathcal{E}_{p}(\rho)\right)$. Figure 1 shows the values of the new distance $D_{N}\left(\rho, \mathcal{E}_{p}(\rho)\right)$ as a function of the parameter $p$, corresponding to different 1-qubit states $\rho$ characterized by different values of the norm of the Bloch vector $\vec{r}$.

\section{SUMMARY AND CONCLUSIONS}

We have studied in detail a new proposal in order to define a metric for quantum states. In the case of two pure states $|\psi\rangle$ and $|\varphi\rangle$, the metric $D_{N}(|\psi\rangle,|\varphi\rangle)$ given in Eq. (11) corresponds to the square root of a von Neumann entropy, more precisely the square root of the quantum Jensen-Shannon divergence. In the case of two mixed states $\rho$ and $\sigma$, the metric $D_{N}(\rho, \sigma)$ given in Eq. (17) is defined as a natural extension of the latter and results to be given as the 
minimum of the distance between pure states corresponding to purifications of both mixed densities. From a physical point of view we have replaced the problem of evaluating the distance between two mixed states by the problem of distinguishing ensembles built from purifications of that states.

We have analyzed some of the properties of the new proposal, justifying its metric character, and illustrated how to implement the minimization procedure with an application for two 1-qubit mixed states. As a particular example, we have considered a depolarizing channel. By using the representation for the purifications of multiple qubits states [23], an evaluation of the distance $D_{N}$ in this case can be also performed.

It should be emphasized that our proposal, although close, differs from another ones. For example the definition of the Shannon distinguishability 25] involves the Shannon entropy and quantum measurement processes. It should be stressed also that, although the computational effort of evaluating the minimum in (17) and (16) could be similar, the metric $D_{N}$ is grounded in two very relevant physical concepts as are entropy and purification. The study of some applications of this metric in the context of decoherence and entanglement is in progress.

\section{Acknowledgments}

This work was partially supported by CONICET (National Research Council), Argentina. PWL wants to thank SECyT-UNC (Argentina) for financial assistance. The authors want to thank Prof. Domingo Prato for useful discussion.

[1] M.A. Nielsen and I.L. Chuang, Quantum Computation and Quantum Information (Cambridge University Press, Cambridge, England, 2000)

[2] V. Vedral, Rev. Mod. Phys. 74, 197-234 (2002)

[3] V. Vedral, M.B. Plenio, K. Jacobs and P.L. Knight, Phys. Rev. A 56, 4452 (1997)

[4] I. Bengtsson and K. Życzkowski, Geometry of Quantum States: An Introduction to Quantum Entanglement (Cambridge University Press, Cambridge, 2006)

[5] W.K. Wootters, Phys. Rev. D 23, 357 (1981)

[6] S.L. Braunstein and C.M. Caves, Phys. Rev. Lett. 72, 3439 (1994)

[7] A. Uhlmann, Rep. Mat. Phys 9, 273-279 (1976)

[8] R. Jozsa, J. Mod. Opt. 41, 2315-2323 (1994)

[9] J. Lee, M.S. Kim and C. Brukner, Phys. Rev. Lett. 91, 087902 (2003)

[10] Z.-H. Ma, F.-L. Zhang and J.-L. Chen, arXiv:0811.3453k1 (2008)

[11] P.E.M.F. Mendonça, R. d. J. Napolitano, M. A. Marchiolli, C.J. Foster and Y.-C. Liang, Phys. Rev. A 78, 052330 (2008)

[12] G. Lindblad, Commun. Math. Phys. 33, 305 (1973)

[13] A.P. Majtey, P.W. Lamberti and D.P. Prato, Phys. Rev. A 72, 052310 (2005)

[14] P.W. Lamberti, A. Majtey, A. Borras, M. Casas and A. Plastino, Phys. Rev. A 77, 052311 (2008)

[15] J. Briët, P. Harremoës and F. Topsøe, arXiv:0806.4472 2 (2008)

[16] A. Majtey, A. Borras, M. Casas, P.W. Lamberti and A. Plastino, Int. J. Quant. Inf. Theory 6, supp01, 715-720 (2008)

[17] T. Cover and J. Thomas, Elements of Information Theory (Wiley, 1990)

[18] A. Wehrl, Rev. Mod. Phys. 50, 221-260 (1978)

[19] R. Jozsa and J. Schlienz, Phys. Rev. A 62, 012301 (2000)

[20] B. Schumacher, Phys. Rev. A 51, 2738 (1995)

[21] W.H. Zurek, Rev. Mod. Phys. 75, 715 (2003)

[22] A. Majtey, P.W. Lamberti, M.T. Martin and A. Plastino, Eur. Phys. J. D 32, 413-418 (2005)

[23] T. Constantinescu and V. Ramakrishna, Q. Inf. Proc. 1, 405-424 (2002)

[24] P.H. Schönemann, Psychometrika 31, 1-10 (1966)

[25] C.A. Fuchs and J. van de Graaf, arXiv:quant-ph/9712042 2 (1997) 